Article

\title{
Analysis, Design, and Implementation of Improved LLC Resonant Transformer for Efficiency Enhancement
}

\author{
Zhenxing Zhao ${ }^{1,2}$, Qianming $X u^{1, *}$, Yuxing Dai ${ }^{1,3}$ and Hanhang Yin ${ }^{1}$ \\ 1 College of Electrical and Information Engineering, Hunan University, Changsha 410082, China; \\ 22006@hnie.edu.cn (Z.Z.); daiyx@hnu.edu.cn (Y.D.); yinhanhang@hnu.edu.cn (H.Y.) \\ 2 College of Electrical Information, Hunan Institute of Engineering, Xiangtan 411100, China \\ 3 College of Physical and Electrical Engineering, Wenzhou University, Wenzhou 325000, China \\ * Correspondence: xqm@hnu.edu.cn; Tel.: +86-731-8882-3964
}

Received: 9 November 2018; Accepted: 22 November 2018; Published: 25 November 2018

check for updates

\begin{abstract}
In battery charging applications, the charger changes its output voltage in a wide range during the charging process. This makes the design of LLC converters difficult to be optimized between the efficiency and the gain range. In this paper, an improved resonant transformer is presented for LLC resonant converter charger to improve the gain adjustment and charger efficiency. The resonant inductance and magnetizing inductance are integrated in the designed LLC transformer, and the magnetizing inductance can be adjusted dynamically with the change of output voltage and load, which is realized by a switch-controlled inductor (SCI) parallel to the secondary winding of transformer. The proposed transformer has $22.4 \%$ reduction in losses under full load conditions compared to conventional solutions. Moreover, the conduction loss and switching loss of LLC resonant tank are reduced by dynamically adjusting the magnetizing inductance, which improves the comprehensive efficiency of the whole charging process. The proposed transformer design is verified on a $720 \mathrm{~W}$ prototype.
\end{abstract}

Keywords: LLC resonant converter; resonant transformer; fringing effect; adjustable magnetizing inductance; efficiency

\section{Introduction}

LLC resonant converter has been widely used in electric vehicle battery chargers, flat panel television (TV), and photovoltaic (PV) system due to its high-power density and high conversion efficiency [1-4]. For now, it has become one of the most concerned DC/DC converters.

In constant output voltage applications, LLC resonant converter can achieve high efficiency. However, there are many challenges for LLC resonant converter in the charger applications which requires a wide output voltage adjustment range [5]. It requires a small magnetizing inductance to obtain a wide output voltage adjustment range, but it can lead to increased conduction loss and switching loss as well as efficiency reduction [6]. The magnetizing inductance is usually integrated in LLC resonant transformer. The usual structure of magnetic integrated LLC transformer is as shown in Figure 1, the leakage layer is set between the primary winding and secondary winding. The required resonant inductance is obtained by using stray flux and the magnetizing inductance is achieved by inserting an appropriate air-gap in the magnetic circuit $[7,8]$. The transformer with that structure has high integration and low cost and has been widely used in converters with power level from dozens to hundreds of watts. 


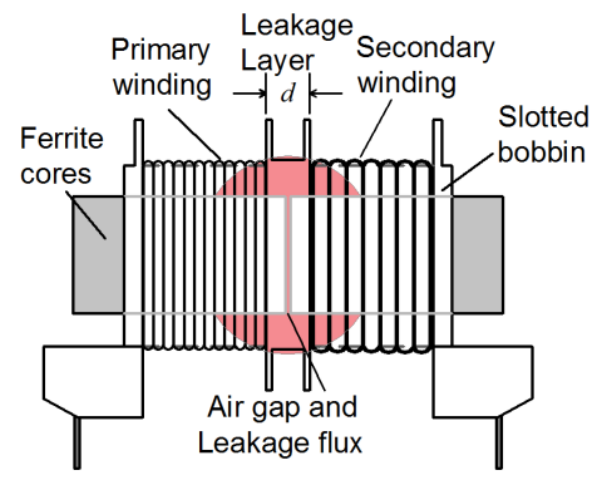

Figure 1. Structure of conventional LLC transformer and leakage flux effect on the windings.

As the core device of LLC resonant converter, the transformer plays a critical role in converter's efficiency, volume, power density, and reliability. However, the whole loss of the LLC resonant transformer has a much higher percentage than that of phase-shift full-bridge transformer in the same power [9]. One of the main reasons is that the air gap in the magnetic core of LLC resonant transformer causes fringing effect, leading to an increase in the equivalent resistance of windings near the air gap, and the transformer inner temperature rise distributed imbalance. The other reason is that the small magnetizing inductance leads to increased conduction loss and switching loss of the LLC resonant converter, so the light-load efficiency is lower.

In order to improve the efficiency of LLC transformer, different improvement designs are proposed. In [10], a novel shape magnetic core is used to achieve the integration of two LLC transformers. In [11], a magneto plated wire is used to effectively decrease the winding loss caused by the proximity effect when working in high frequency. In [12], the integration of resonant inductance required is achieved by inserting a layer of flexible magnetic material between the primary and secondary windings. The designs proposed by [10-12] have high integration, but without regard for the fringing effect, so they are more suitable for low power applications. In [13], the copper loss of the Litz wire caused by the air gap is analyzed, and the influence of the fringing effect is weakened by using multiple small air gaps in series, but this increases the cost and difficulty of transformer production. In [7], a design method of LLC resonant transformer is presented, and the current density in the conductor near the air gap is simulated. In [14], the optimal design method of the transformer in conventional LLC resonant converter is extended to the design and application of flat panel transformer, and a complete design scheme and a detailed application method are given. In [7,14], the fringing effect is mentioned, but the influence of the effect is not analyzed and the solution is not given. In [15], the relationship between the temperature rise and the switch frequency as well as the winding number of the LLC resonant transformer are discussed, and a combination of two smaller transformers is used instead of one larger transformer to reduce the temperature rise. Matrix transformer is used to achieve a higher output power in [16]. However, with the increase of power, the matrix transformer which consists of too many transformers will lead the system more complex. In [17,18], to reduce the influence of the fringing effect, the distance between the winding and the air gap is increased by making changes of the winding structure, which leads to the increase in difficulty of processing windings.

To improve the light-load efficiency, the transformers in $[19,20]$ are designed with variable magnetizing inductance. In [19], by using utilizing a step-gap in the core column, a larger magnetizing inductance is obtained at light load, and it decreases at heavy load, but the variety of magnetizing inductance is non-linear and uncontrollable. In [20], a bidirectional power switch is used to achieve the parallel operation of two transformers. When under heavy load, the switch turn-on, two transformers primary side work in parallel, the magnetizing inductance of resonant tank is small, and can satisfy the gain demand required in the initial stage of charging. When under light load, the switch turns off, only one transformer works, the magnetizing inductance of resonant tank is large, and the conduction loss and switching loss of primary side decrease. However, the resonant inductance cannot be integrated 
into the transformer, the magnetizing inductance can only be changed between two fixed values, and when under light load, only one transformer works and the other is vacant.

Aiming to address the design problems of the conventional LLC transformer caused by the large influence of the fringing effect and the unchangeable magnetizing inductance, this paper presents a design for the integrated LLC resonant transformer. The magnetizing inductance does not need to be obtained by inserting air gap, so the fringing effect of the transformer is small. Moreover, the magnetizing inductance can be dynamically adjusted by a SCI parallel to the secondary winding of the transformer. This can not only improve the efficiency and the temperature rise of the transformer but also optimize the comprehensive efficiency of the LLC resonant converter during the whole charging process.

This paper includes following aspects. Section 2 analyzes the conventional integrated LLC resonant transformer. Section 3 presents the proposed design scheme, analysis of magnetic flux in the transformer core and the application circuit. Section 4 gives the calculation formula and design procedure of the transformer. Section 5 shows the experimental results of a $720 \mathrm{~W}$ LLC resonant converter. The conclusion is given in the last section.

\section{Analysis of Conventional LLC Transformer}

In high-power applications, the fringing effect near the air gap leads to a significant increase in the current density of the windings near the air gap, resulting in an apparent increase in the partial temperature rise and copper loss of the transformer [7]. To analyze the influence of fringing effect, finite element analysis (FEA) simulation is carried out by using Maxwell software. Figure 2 illustrates the simulation of fringing effect at $100 \mathrm{kHz}$. It can be seen the current density of the copper conductor near the air gap is about $70 \%$ higher than that away from the air gap, so the copper loss of this part is significantly increased. The increased copper loss results in the conspicuous regional temperature rise near the air gap. The simulation also shows that the fringing effect has a significant impact near the air gap, and the affected range increases with the increase of transformer power from the center of the air gap.

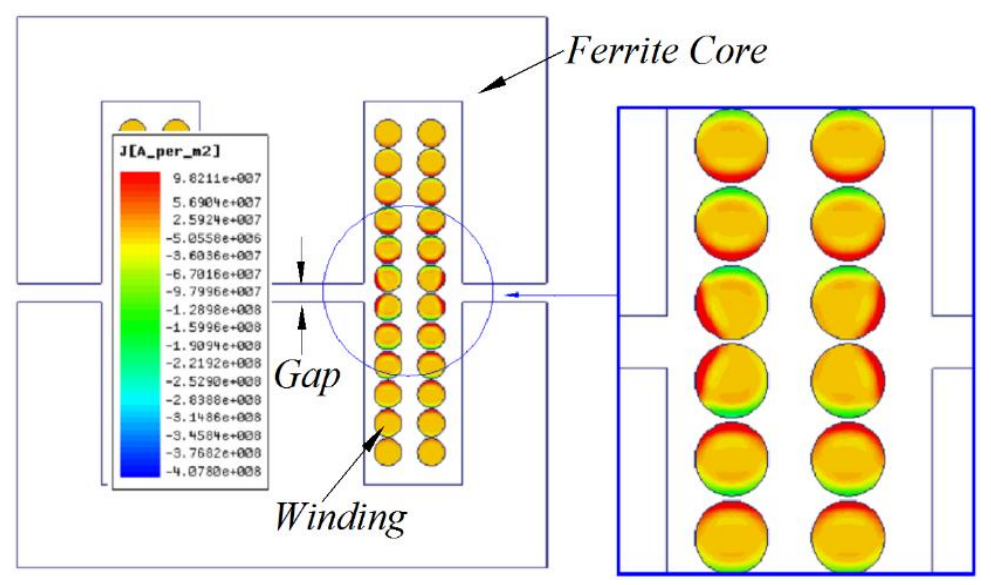

Figure 2. High frequency fringing effect near the air gap.

After the winding turns and air gap size of the transformer are determined, the transformer magnetizing inductance is a fixed value. Choosing small magnetizing inductance to obtain sufficient output voltage gain will result in increased conduction loss and switching loss of the LLC resonant converter.

The simulation results show that the induced magnetic flux increases in pace with the increase of the transformer power and input voltage, the area influenced by the air gap expand accordingly. In order to reduce the fringing effect, it is needed to increase distance $d$ of leakage layer, as shown in Figure 1 . However, the increasing $d$ causes the available area of the core window decrease, under the 
same diameter and turns, the layer $p$ of transformer winding is increase. According to the Dowell's formula as below, this effect can lead to a significant increase in proximity loss.

$$
\frac{R_{a c}}{R_{d c}}=\Delta\left[\frac{\sinh 2 \Delta+\sin 2 \Delta}{\cosh 2 \Delta-\cos 2 \Delta}+\frac{2\left(p^{2}-1\right)}{3} \frac{\sinh \Delta-\sin \Delta}{\cosh \Delta+\cos \Delta}\right]
$$

where, $p$ is the number of transformer windings layers, $\Delta$ is the ratio of the winding layer thickness $d$ of the skin depth $\delta_{0}$.

To integrate the resonant inductance into the transformer, the primary winding and the secondary winding cannot use 'sandwich' method, which is used in full-bridge phase-shifting transformer to decrease the proximity loss. Therefore, at high frequency condition, use Litz wire to decrease the impact of the proximity effect and the skin effect.

In summary, the fringing effect, proximity effect, and the increase of the conduction loss and switching loss caused by small magnetizing inductance are main factors affect the transformer efficiency.

\section{Analysis of Improved LLC Resonant Transformer}

In order to promote the efficiency, an improved LLC transformer design is presented.

\subsection{Main Structure}

The structure of proposed LLC resonant transformer is shown in Figure 3, which has the following characteristics. (1) The skeleton adopts double groove structure, thus primary winding and secondary winding are wound respectively in two grooves. Therefore, the resonant inductance is still integrated in the transformer. (2) The air gap is not inserted in the magnetic circuit, so there is nearly no leakage flux, the intensive windings in the transformer is almost not affected by the fringing effect. (3) The thickness $d$ of the middle leakage layer can be small, which does not occupy the space of the windings and improves the utilization rate of the core window, reducing the proximity effect.

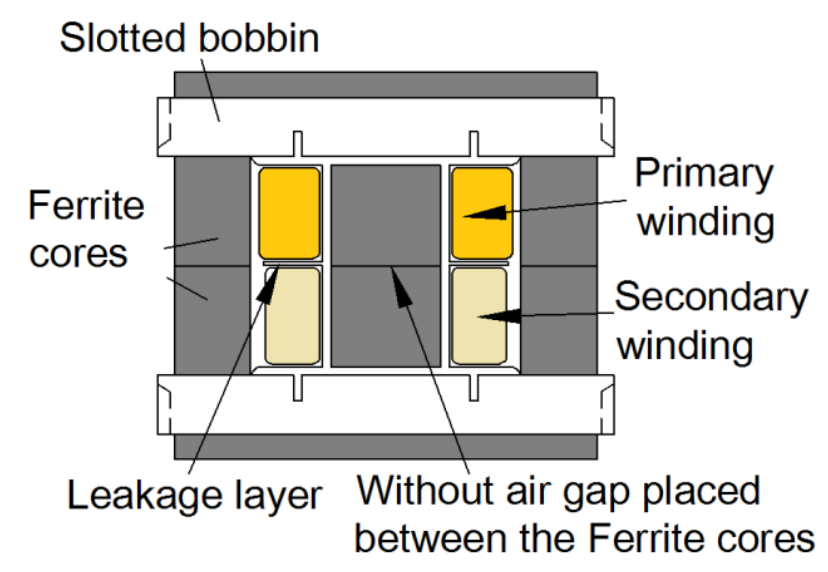

Figure 3. Main structure of proposed transformer.

The fringing effect simulations comparison is shown in Figure 4. It can be seen that the conductor current density near the air gap of the proposed transformer is greatly reduced, and the conduction loss is effectively decreased, so the internal temperature rise can be improved. 


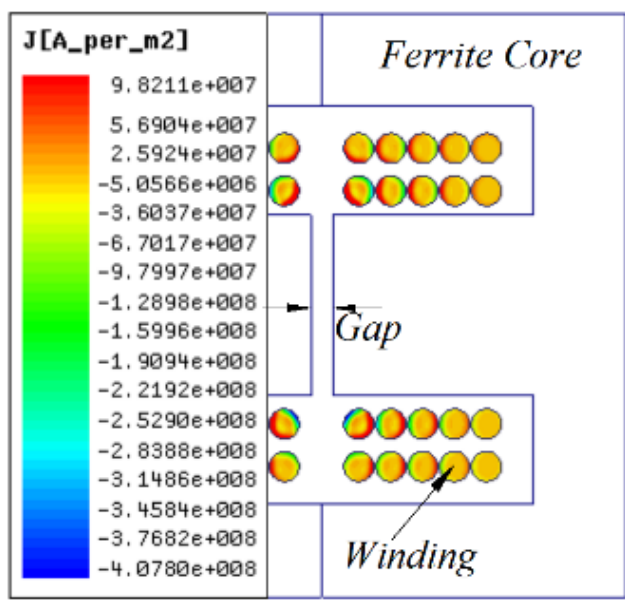

(a)

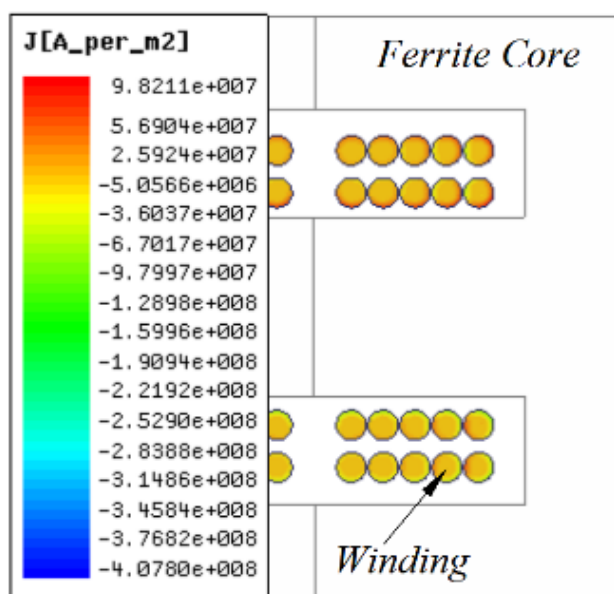

(b)

Figure 4. Fringing effect comparison: (a) Conventional scheme; (b) Proposed scheme.

However, the proposed transformer almost has no air gap, and the equivalent magnetic permeability of the magnetic core is large. Hence, its magnetizing inductance is much larger than that of conventional LLC transformer with air gap, which cannot satisfy the LLC resonant tank demand. For this reason, a SCI is parallel to the secondary winding to achieve the magnetizing inductance adjustment, as shown in Figure 5, Co is the output filter capacitor, and $R_{L}$ is the load.

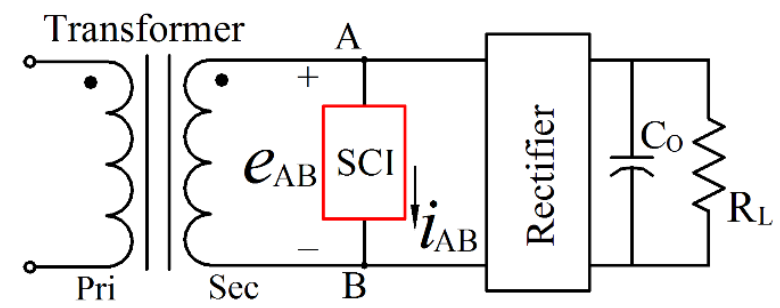

Figure 5. Switch controlled inductor parallel to secondary winding of transformer.

\subsection{Switch Controlled Inductor (SCI)}

The SCI in Figure 5 adopts series type [21], and its circuit structure and working waveforms are shown in Figure 6. The SCI consists of linear inductance $L a$ and two control switches $T a$ and $T b$. The voltage $e_{A B}$ added to the both sides of $\mathrm{SCI}$ is the transformer secondary winding voltage close to square wave. $\mathrm{V}_{\mathrm{Ta}}$ and $\mathrm{V}_{\mathrm{Tb}}$ are the drive signal of switch devices $T a$ and $T b$ respectively, and their frequencies are equal to the operating frequency of the LLC resonant converter. The current flowing through the SCI is $i_{A B} . \delta$ is the phase angle of the switch drive signal. The working principle of SCI in a switch period is analyzed as follows.

At $t_{0}$, when the secondary winding voltage $e_{A B}$ is positive polarity and $T a$ is turn-off, the inductor current flow through SCI is zero. At $t_{1}$, the drive signal makes $T a$ turning on and $T b$ turning off, and $i_{A B}$ increases linearly from zero. Therefore, $T a$ turns on at zero current and $T b$ turn off at zero current. At $t_{2}$, when the polar of $e_{A B}$ reverses, the inductor current $i_{A B}$ reaches a maximum and then begins to decrease linearly. At $t_{3}$, the inductor current $i_{A B}$ decreases to zero. Because of the reverse blocking of $T b, i_{A B}$ remains zero until $t_{4}$. Therefore, $T a$ realizes zero current turn-off and $T b$ realizes zero current turn-on. The opposite direction is the same. Since the conduction resistance of $T a$ and $T b$ is very small, the switching loss and conduction loss of switch devices $T a$ and $T b$ are relatively small. 


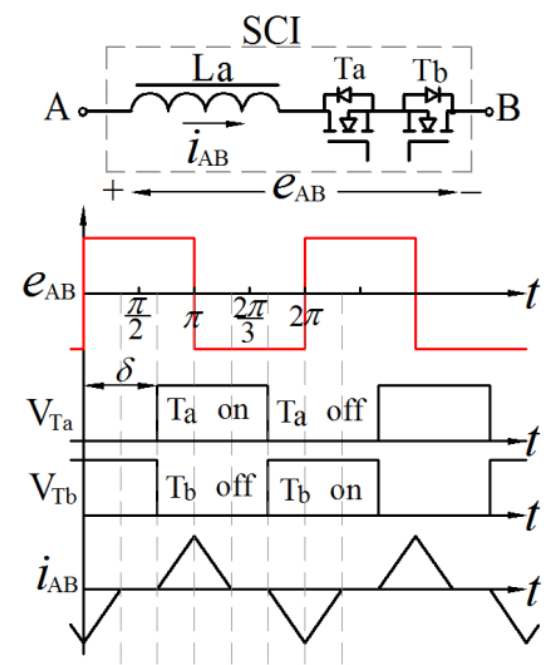

$t_{0} t_{1} t_{2} t_{3} t_{4} t_{5} t_{6}$

Figure 6. Topology and waveforms of SCI.

The equivalent inductance of SCI can be obtained from [22] as Equation (2)

$$
L_{S C I}=\frac{L_{a}}{2-(2 \delta-\sin 2 \delta) / \pi}
$$

According to Equation (2), when the phase angle of switch drive signal ranges between $\pi / 2$ and $\pi$, the equivalent switch-controlled inductance $L_{S C I}$ ranges between $L a$ and $\infty$. The relationship curve between inductance ratio $L_{S C I} / L_{a}$ and the phase angle of switch drive signal is shown in Figure 7 . It can be found the circuit shown in Figure 5 can be equivalent to an inductance which can be adjusted from $L a$ to $\infty$.

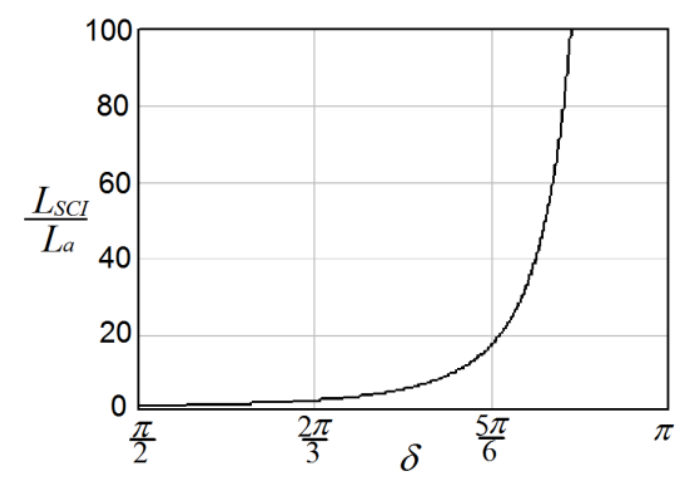

Figure 7. Equivalent inductance of SCI.

\subsection{Equivalent Circuit of Resonant Tank and Magnetic Field Analysis}

The equivalent circuit of LLC resonant tank for secondary winding parallel SCI is shown in Figure 8. where $C_{r}$ is the resonant capacitance, $L_{r}$ is the resonant inductance and $L_{m \text {-ini }}$ is the large magnetizing inductance integrated in transformer, as shown in Equation (3).

$$
L_{m-i n i}=\frac{\mu_{e f f} \cdot N_{p}^{2} \cdot A_{c}}{l_{c}}
$$

where $\mu_{e f f}$ is the equivalent magnetic permeability of the magnetic circuit approximately equal to the relative permeability of the core material $\mu_{r} . N_{p}$ is the turns of primary winding, $A_{c}$ is the cross-section area of the magnetic core, $l_{c}$ is the magnetic circuit length. Since $\mu_{e f f} \approx \mu_{r}$, the value of $L_{m-i n i}$ is large as result of the large relative permeability of the core material. 


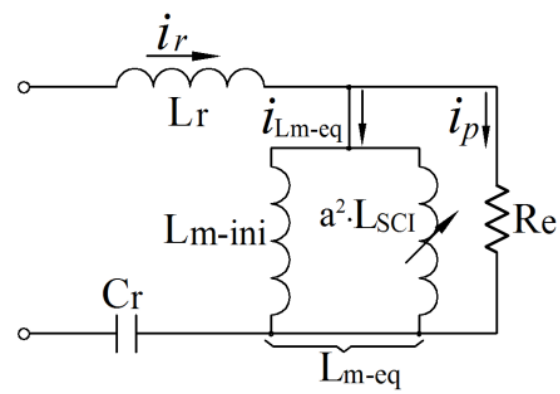

Figure 8. Equivalent circuit of the proposed LLC resonant tank.

Setting $a$ is the ratio of the transformer, and $a^{2} \cdot L_{S C I}$ is the primary equivalent inductance reflected by the SCI. $R e$ is the equivalent load impedance, and $R_{\text {load }}$ is the load resistance. The magnetizing inductance in the resonant tank can be expressed as Equation (4).

$$
\begin{aligned}
L_{m-e q} & =L_{m-i n i} \| a^{2} \cdot L_{S C I} \\
R_{e} & =\frac{8}{\pi^{2}} \cdot a^{2} \cdot R_{\text {load }}
\end{aligned}
$$

Due to the adjusted value of $L_{S C I}$ driven by $\delta$, so the inductance $a^{2} \cdot L_{S C I}$ in Equation (4) can be represented as an adjustable inductance. Therefore, the equivalent magnetizing inductance $L_{m-e q}$ can also be adjusted by changing $\delta$.

As shown in Figure 8, when the resonant tank is transferring energy to secondary side, the resonant frequency is $f_{r}$.

$$
f_{r}=\frac{1}{2 \pi \sqrt{L_{r} C_{r}}}
$$

Otherwise, the magnetizing inductance $L_{m-e q}$ participates in resonant, and the resonant frequency is Equation (7).

$$
f_{r}^{\prime}=\frac{1}{2 \pi \sqrt{\left(L_{r}+L_{m-e q}\right) C_{r}}}
$$

Inner magnetic flux analysis of the transformer is shown in Figure 9. The primary side is on the left, and the secondary side is on the right. The flux $\phi_{\mathrm{p}}$ and $\phi_{S}$ in the magnetic core established respectively by the primary resonant current $i_{p}$ and the secondary current $i_{s}$ cancel each other. Meanwhile, the flux $\phi_{L m-e q}$ and $\phi_{A B}$ in the magnetic core established respectively by the magnetizing current $i_{L m-e q}$ and the SCI current $i_{A B}$ cancel each other. It follows that, the transformer does not store energy, and the inductive energy storage required by LLC half-bridge switch for zero voltage switching (ZVS) is completed by SCI.

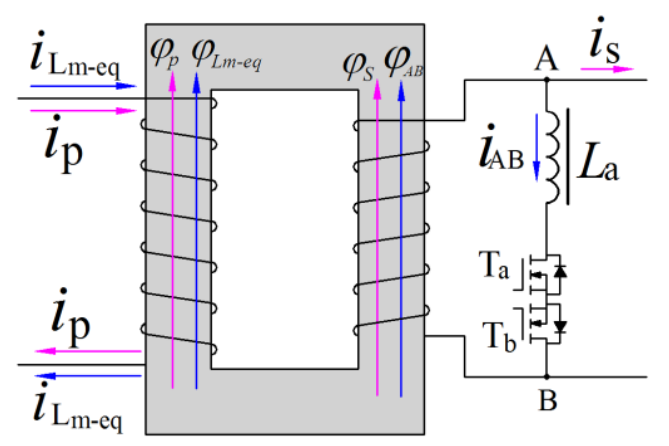

Figure 9. Magnetic flux in the core of transformer. 


\subsection{LLC Resonant Converter Charger Application}

The application of the proposed transformer in LLC resonant converter charger is shown in Figure 10, and the secondary side adopts a full-bridge rectifier. The resonant tank consists of $C_{r}, L_{r}$, and $L_{m-e q}$.

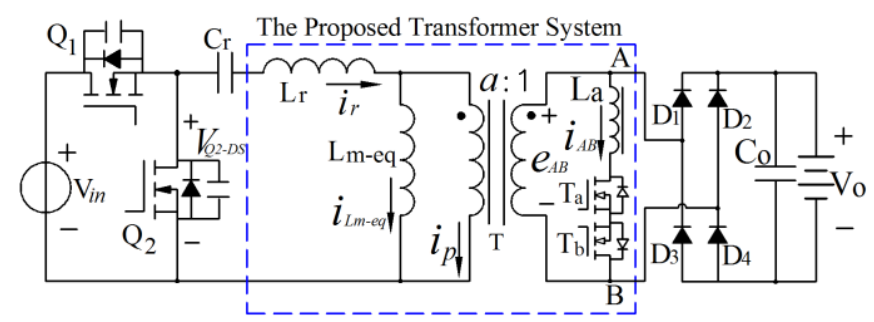

Figure 10. Proposed resonant transformer applied to LLC resonant converter charger. (8).

The ratio of the equivalent magnetizing inductance to resonant inductance is defined as Equation

$$
\begin{gathered}
k=\frac{L_{m-e q}}{L_{r}} \\
Q=\frac{\sqrt{L_{r} / C_{r}} \cdot \pi^{2}}{8 \cdot a^{2} \cdot R_{\text {load }}}
\end{gathered}
$$

In Equation (9), $Q$ is the quality factor. Under constant $L_{r}, C_{r}$, and $a, Q$ is only related to load. Under the same load ( $Q$ value), the voltage gain curves with different $k$ are shown in Figure 11. The horizontal axis is the normalized frequency, which is the ratio of working frequency $f_{s}$ to resonant frequency $f_{r}$, and the vertical axis is DC voltage gain. It can be seen that in the same frequency range, a resonant tank with smaller $k$ can get a larger output voltage regulating range. Moreover, under the condition of fixed resonant inductance $L_{r}$, the proposed transformer can regulate $L_{m-e q}$ to adjust $k$, so that it can dynamically adjust the voltage gain of LLC resonant converter in the charging process according to the corresponding demand.

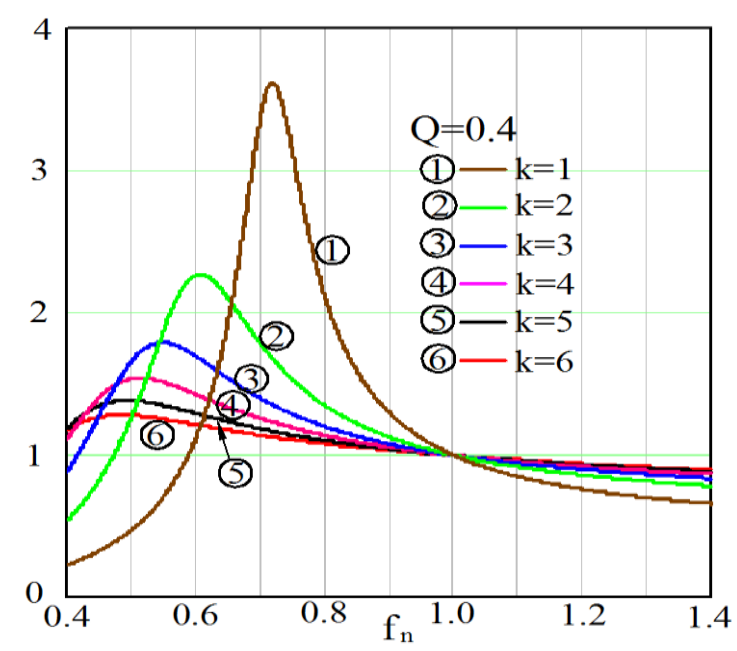

Figure 11. Gain curves with different $k$ value.

Furthermore, the simplified typical battery charging curve is shown in Figure 12. The charging process can be divided to three stages: constant current charging, constant power charging, and constant voltage charging. The output power becomes very small at the charging end.

$$
Q=\frac{\sqrt{L_{r} / C_{r}} \cdot \pi^{2} \cdot I_{o}}{8 \cdot a^{2} \cdot V_{o}}
$$


At the initial stage of charging, the charger current $I_{o}$ is large, and the output voltage $V_{o}$ is relatively small. At the latter stage of charging, the output voltage $V_{o}$ is relatively high and the output current $I_{o}$ is small. According to Equation (10), the $Q$ value should be large at the initial stage of charging. With the charging process carrying on, the $Q$ value decreases. Figure 13 gives several gain curves of $k$ value in the charging process. The shaded area (ZVS region) in Figure 13 is the work areas of LLC resonant converter. Usually, the operating frequency of LLC resonant converter is set as the resonant frequency with the maximum output power (point A in Figure 12), since the LLC converter can get highest efficiency [22].

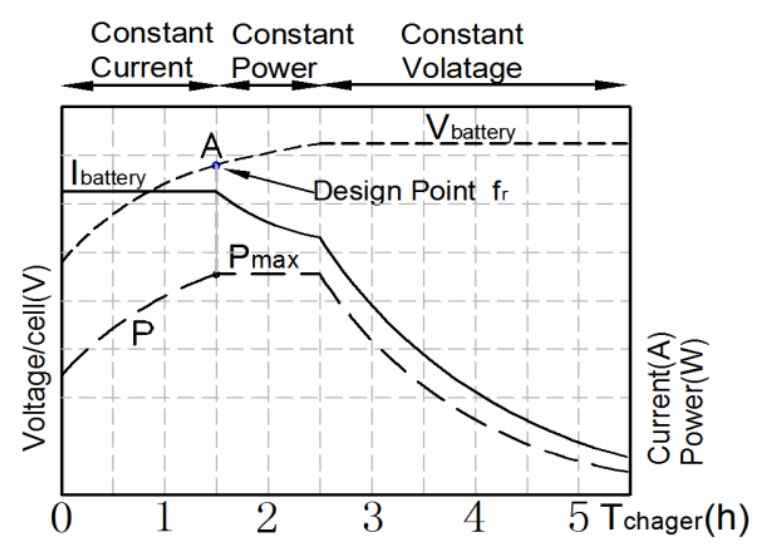

Figure 12. Simplified battery charging profile.

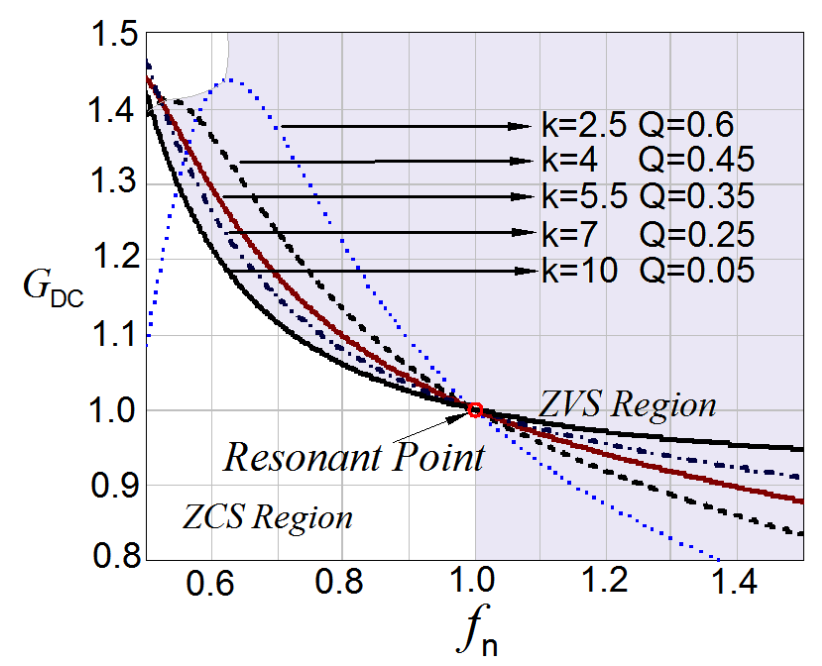

Figure 13. Change of gain curve during charging process.

Therefore, the operating frequency of the constant current charging stage in Figure 12 is located at the right of the resonant frequency $\left(f_{n}=1\right)$ in Figure 13. The primary switching device of LLC resonant converter works in the ZVS conduction state, but the turn-off loss increases with the increase of frequency and the secondary rectifier diode loses ZCS characteristic. Therefore, in this area, the operating frequency can be set as close as possible to the resonant frequency. As shown in Figure 13, the curve corresponding to a smaller $\mathrm{K}$ value is closer to the resonant frequency point (the frequency range is smaller with same gain range). When charging process enters constant power stage, the LLC resonant converter works at the left of the resonant frequency point. The primary switch of LLC resonant converter can realize ZVS conduction, and the secondary rectifier diode can realize ZCS turn-off, which can achieve better efficiency. With the decrease of operating frequency, the smaller $\mathrm{K}$ value leads to the increased resonant tank magnetizing current, conduction loss as well as turn-off loss. Especially, when at light load, the proportion of conduction loss in the whole loss increases and reduces 
overall efficiency. Therefore, with the decrease of output power and the increase of output voltage, the magnetizing inductance should gradually increase ( $k$ value gradually increases). In the conventional scheme, the $\mathrm{K}$ value is constant after the magnetizing inductance is selected. However, the proposed transformer can make the LLC resonant converter change its equivalent magnetizing inductance according to load and output voltage during the whole charging process, so that the gain characteristic, circulation loss, and switching loss of LLC resonant tank can be optimized via programming.

\section{Design Methodology}

\subsection{Electrical Design Considerations}

A: Selection of magnetizing inductance

The maximum magnetizing inductance to realize ZVS under idle condition is $L_{m 1}$, and the maximum value to ensure the maximum gain at the lowest frequency is $L_{m 2}$. The final value of the magnetizing inductance should satisfy the above two requirements at the same time. Hence, the smaller one between $L_{m 1}$ and $L_{m 2}$ can be chosen. If $L_{m 1}$ is greater than $L_{m 2}$, the dead time $t_{\text {dead }}$ should be appropriately reduced [23], as shown in Equation (11).

$$
\left\{\begin{array}{l}
L_{m 1}=\frac{t_{\text {dead }} \cdot a \cdot V_{o(\min )} \cdot\left(\frac{T_{s(\min )}}{4}-\frac{t_{\text {dead }}}{2}\right)}{C_{H B} \cdot V_{i n(\max )}} \\
L_{m 2}=L_{r} \cdot \frac{\pi^{2}}{4} \cdot \frac{1-\frac{1}{G} \cdot \frac{1}{f_{r}(\max )}}{f_{s(\max )}-1} \\
L_{m-e q}=\min \left(L_{m 1}, L_{m 2}\right)
\end{array}\right.
$$

where $G_{D C(\max )}$ is the maximum $D C$ gain, and $C_{H B}$ is the total equivalent capacitance of the $\mathrm{H}$ bridge.

B: Selection of minimum resonant inductance

The minimum resonant inductance should limit the maximum output current under short circuit when working at the highest frequency.

$$
L_{r(\min )}=\frac{a \cdot V_{i n(\text { norm })} \cdot V_{o(\text { norm })}}{8 f_{s(\max )} \cdot P_{o}}
$$

C: Selection of resonant capacitance

After $L_{r}$ is selected, the resonant capacitance can be obtained by Equation (13).

$$
C_{r}=\frac{1}{\left(2 \pi \cdot f_{r}\right)^{2} L_{r}}
$$

\subsection{Transformer Loss and Thermal Design Considerations}

The loss of transformer includes core loss and winding loss.

A: Core loss

The core loss and core loss can be calculated by Steinmetz formula.

$$
P_{f e}=V_{c} K_{c} f^{\alpha} B_{\max }^{\beta}
$$

where $V_{c}$ is the core volume, $K_{c}$ is the typical value. $\alpha$ and $\beta$ are provided by core manufacturer or obtained by loss curve. 


\section{B: Winding loss}

The winding loss include DC loss and AC loss. The current through the winding can be calculated as below.

The current through the primary winding $i_{r}$ is the sum of $i_{p}$ and $i_{L m-e q}$, and the current through secondary winding is the sum of $i_{S}$ and $i_{A B}$. The expressions of the peak current through primary winding is $I_{r-p e a k}$ with the RMS value $I_{r-r m s}$, and the peak magnetizing current $I_{L m-\text { peak }}$ are [7]

$$
\begin{gathered}
I_{r-\text { peak }}=\sqrt{\left(\frac{\pi \cdot I_{o}}{2 a f_{n}}\right)^{2}+\left(\frac{a V_{o}}{4 f_{r} L_{m-e q}}\right)^{2}} \\
I_{L m-\text { peak }}=\frac{a V_{o}}{4 L_{m-e q} f_{r}} \\
I_{r-r m s}=\sqrt{\frac{a^{2} V_{o}^{2} T_{r}^{2}\left(2 T_{s}-T_{r}\right)}{32 L_{m}^{2} T_{s}}+\frac{\pi^{2} I_{o}^{2} T_{s}^{2}}{8 a^{2} T_{r}^{2}}}
\end{gathered}
$$

where $T_{S}$ and $T_{r}$ are the switching period and resonant period respectively, $V_{o}$ and $I_{o}$ are the output voltage and output current, respectively.

By using Equation (18), the RMS value of the current though secondary winding can be obtained.

$$
i_{S_{-} r m s}=\sqrt{\frac{2 \cdot \int_{0}^{\frac{T_{r}}{2}}\left(a I_{r_{-} p e a k} \sin \left[\omega_{r} t+\phi\right]+\frac{a^{2} V_{o}}{4 L_{m} f_{r}}-\frac{a^{2} V_{o}}{L_{m}} t\right)^{2} d t}{T_{r}}}
$$

where $\omega_{r}=2 \pi f_{r}, \phi=\arctan \left(-\frac{a^{2} R_{L} f_{s}}{\omega_{r} L_{m} f_{r}}\right)$.

The DC copper loss is calculated as below.

$$
P_{c u}=R_{p-r m s} I_{r-r m s}^{2}+R_{s-r m s} I_{s-r m s}^{2}
$$

The AC impedance of winding can be got from Equation (1).

C: Temperature rise consideration

The maximum loss is determined by the core thermal resistance and the permissible temperature increase.

$$
P_{L \_\max } \approx \frac{\Delta T}{R_{\theta}}
$$

where $R_{\theta}$ is the thermal resistance of the core provided by manufacturer or obtained from empirical data, $h_{c}$ is thermal conductivity, and $A_{t}$ is the surface area of transformer.

$$
R_{\theta}=\frac{1}{h_{\mathrm{c}} A_{t}}
$$

\subsection{Transformer Design Considerations}

Under the premise of minimize core loss and winding loss, the design purpose of a transformer is to transfer energy from input side to output side by electromagnetic induction. The optimization result can be boiled down to one conclusion: iron loss is equal to copper loss [23]. The transformer design method is related to the best magnetic induction intensity and temperature rise of magnetic core and is limited by the maximum permissible power loss. 


\section{A: Core selection}

The appropriate core is up to $A_{p}$ value. $A_{p}$ is the product of core window area $W_{a}$ and core cross-section area $A_{c}$, as Equation (22).

$$
A_{p}=\left(\frac{\sqrt{2} \sum V A}{K_{v} f_{s} B_{o} k_{f} K_{t} \sqrt{k_{u} \Delta T}}\right)^{\frac{8}{7}}
$$

where, $\sum V A$ is the sum of each windings rated $V A$ values, $K_{v}=4.44, f_{s}$ is the operating frequency, $B_{0}$ is the best magnetic induction intensity value, $K_{t}=48.2 \times 103, k_{f}$ is the core lamination factor, $A_{m}$ is the effective sectional area of magnetic circuit.

The best magnetic induction intensity $B_{0}$ is given by Equation (23).

$$
B_{0}=\frac{\left(h_{c} k_{a} \Delta T\right)^{\frac{2}{3}}}{2^{\frac{2}{3}}\left(\rho_{w} k_{w} k_{u}\right)^{\frac{1}{12}}\left(k_{c} K_{C} f^{\alpha}\right)^{\frac{7}{12}}}\left(\frac{K_{v} f k_{f} k_{u}}{\sum V A}\right)^{\frac{1}{6}}
$$

where, $h_{c}$ is the thermal convection transfer coefficient with typical value 10 , and $k_{a}, k_{c}$, and $k_{w}$ are dimensionless constants with the typical values $k_{a}=40, k_{c}=5.6, k_{w}=10 . K_{C}, \alpha$ are the material parameters, $\rho_{w}$ is the wire resistivity.

B: Calculation of transformer winding turns and turns ratio

The winding turns can be calculated by Equation (24).

$$
N=\frac{V_{r m s}}{K_{v} f B_{\max } A_{m}}
$$

where $V_{r m s}$ is the wingding terminal voltage, $A_{m}$ is the core cross-section area, $B_{\max }$ is the smaller one between $B_{o}$ and $B_{\text {sat }}$. $B_{o}$ is usually smaller than $B_{\text {sat }}$ at the high frequency condition.

The turns ratio can be calculated by Equation (25). where, $V_{d}$ is the conduction voltage drop of the secondary rectifier diode.

$$
a=\frac{V_{\text {in }(\text { norm })}}{2\left(V_{o(\min )}+V_{d}\right)}
$$

C: Transformer wire diameter selection

The current density $J_{0}$ in the wire should satisfy the temperature rise requirement under the whole power loss.

$$
J_{0}=K_{t} \frac{\sqrt{\Delta T}}{\sqrt{k_{u}(1+\gamma)} \sqrt[8]{A_{p}}}
$$

The calculation of wire sectional area $A_{w}$ is Equation (27).

$$
A_{w}=\frac{I_{r m s}}{J_{0}}
$$

D: Determination of inductance La

According to Equation (2) and Figure 7, the switch-controlled inductance $\mathrm{L}_{\mathrm{SCI}}$ can be adjusted in a wide range. When $\alpha=\pi / 2, L_{S C I}=L a$. While the $k$ value of LLC resonant tank obtains a minimum value $k_{\min }$ as Equation (28) According to Equation (4).

$$
L_{a}=\frac{k_{\min } \cdot L_{r} \cdot L_{m-i n i}}{a^{2}\left(L_{m-i n i}-k_{\min } \cdot L_{r}\right)}
$$




\section{Experimental Verification}

\subsection{Design Specification}

An LLC resonant DC/DC converter with $720 \mathrm{~W}$ output power is designed, its input voltage is $390 \mathrm{VDC}$ and output voltage is $60 \mathrm{VDC}-96 \mathrm{VDC}$ with maximum output current is $8 \mathrm{~A}$. The resonant frequency is $85 \mathrm{kHz}$. According to Equation (12), the resonant inductance $L_{r}$ should be greater than $44 \mu \mathrm{H}$ and set as $50 \mu \mathrm{H}$. According to Equation (13), the resonant capacitance $C_{r}$ is $70 \mathrm{nF}$. According to Equation (24), the transformer turns ratio $a=2: 1$. According to Equation (28), $k_{\min }$ is $3, L_{\min }$ is $1 \mathrm{mH}$ and $L a=44 \mu \mathrm{H}$.

The design requirement is shown in Table 1, the RMS value of resonant current, the peak magnetizing current and the RMS value of secondary current are calculated by Equations (15)-(18). The core window utilization factor $k_{u}$ is 0.55 .

Table 1. Design Specification.

\begin{tabular}{ccc}
\hline Symbol & Description & Value \\
\hline$P_{o}$ & Output power & $760 \mathrm{~W}$ \\
$f_{s}$ & Switching frequency & $50 \mathrm{k}-300 \mathrm{kHz}$ \\
$f_{\mathrm{r} 1}$ & Resonant frequency & $85 \mathrm{kHz}$ \\
$L_{a}$ & Magnetizing inductance & $44 \mu \mathrm{H}$ \\
$L_{r}$ & Resonant inductance & $50 \mu \mathrm{H}$ \\
$a$ & Turns ratio & $2: 1$ \\
$\Delta T$ & Temperature rise & $70^{\circ} \mathrm{C}$ \\
$T_{a}$ & Ambient temperature & $40^{\circ} \mathrm{C}$ \\
$I_{r-r m s}$ & Resonant rms current & $4.53 \mathrm{~A}$ \\
$I_{L m-p e a k}$ & Magnetizing peak current & $1.26 \mathrm{~A}$ \\
$I_{S-r m s}$ & Secondary rms current & $9.1 \mathrm{~A}$ \\
$I_{A B-r m s}$ & SCI current & $2 \mathrm{~A}$ \\
$\sum V A$ & The total rated VA values of windings & $1466.3 \mathrm{VA}$ \\
\hline
\end{tabular}

\subsection{Transformer Parameters}

The ferrite core is used for the high switch frequency. $B_{0}=0.1 \mathrm{~T}$ is calculated by Equation (23), $A_{p}=2.5 \mathrm{~cm}^{4}$ is calculated by Equation (22), the ETD44 magnetic core is chosen. The magnetic material is the N87 from EPCOS company, the parameters of this material are given in Table 2, and the parameters of core and winding are given in Table 3. The saturation flux density of this material is $0.4 \mathrm{~T}$. By Equation (24), the transformer secondary turns can be calculated as $N s=11.9 \approx 12$. The primary turns number is 24 .

Table 2. Material Specifications (Epcos N87).

\begin{tabular}{ccc}
\hline Symbol & Description & Value \\
\hline$K_{c}$ & Steinmetz parameter & 16.9 \\
$\alpha$ & Steinmetz parameter & 1.25 \\
$\beta$ & Steinmetz parameter & 2.35 \\
$B_{s a t}$ & Saturation magnetic flux & $0.4 \mathrm{~T}$ \\
$k_{u}$ & Window utilization factor & 0.55 \\
$A_{m}$ & Effective magnetic circuit sectional area & $213 \mathrm{~mm}^{2}$ \\
$\gamma$ & Ratio of core loss to winding loss & 1 \\
\hline
\end{tabular}


Table 3. Core and Winding Parameters.

\begin{tabular}{ccc}
\hline Symbol & Description & Value \\
\hline$A_{c}$ & Cross-section area & $1.73 \mathrm{~cm}^{2}$ \\
$l_{c}$ & Magnetic path length & $10.3 \mathrm{~cm}$ \\
$W_{a}$ & Window area & $2.78 \mathrm{~cm}^{2}$ \\
$A_{p}$ & Area product parameter & $4.81 \mathrm{~cm}^{4}$ \\
$V_{c}$ & Volume of core & $17.70 \mathrm{~cm}$ \\
$M L T$ & Mean length of a turn & $7.77 \mathrm{~cm}$ \\
$k_{f}$ & Core stacking factor & 1.0 \\
$\rho_{20}$ & Copper resistivity $\left(20^{\circ} \mathrm{C}\right)$ & $1.72 \mu \Omega \cdot \mathrm{cm}$ \\
$\alpha_{20}$ & Constant & 0.00393 \\
\hline
\end{tabular}

The current density can be obtained by Equation (26) as $344 \mathrm{~A} / \mathrm{cm}^{2}$, and the wire sectional area of the primary winding is $0.0132 \mathrm{~cm}^{2}$ from Equation (27). Considering the wire skin effect at high frequency, the skin depth is

$$
\lambda_{0}=\frac{66}{\sqrt{f_{s}}}
$$

It is set as $\lambda_{0}=0.226 \mathrm{~mm}$ and the specification parameters are shown in Table 4 . The DC resistance of primary winding is $120.3 \mu \Omega / \mathrm{cm}\left(20^{\circ} \mathrm{C}\right)$.

Table 4. Core and Winding Parameters.

\begin{tabular}{cccc}
\hline AWG & Sectional Area & Resistivity & Diameter \\
\hline 24 & $0.2047 \mathrm{~mm}^{2}$ & $842.1 \mu \Omega / \mathrm{cm}$ & $0.51 \mathrm{~mm}$ \\
\hline
\end{tabular}

To reduce the secondary proximity effect, the Litz wire of $0.15 \mathrm{~mm} \times 110 \mathrm{~mm}$ is chosen. The equivalent electric conduction area is $1.95 \mathrm{~mm}^{2}$, the current density is $464 \mathrm{~A} / \mathrm{cm}^{2}$, and the primary DC internal resistance is $89.2 \mu \Omega / \mathrm{cm}\left(20^{\circ} \mathrm{C}\right)$.

The DC internal resistance of each winding can be obtained with temperature correction as

$$
R_{c u-d c}=N \cdot M L T \cdot \rho_{w} \cdot\left[1+\alpha_{20}\left(T_{\max }-20^{\circ} \mathrm{C}\right)\right]
$$

where $T_{\max }$ is the maximum temperature.

Considering the wire skin effect at high frequency, the skin effect factor is calculated by

$$
\frac{R_{a c-p r i}}{R_{d c-p r i}}=1+\frac{\left(r_{o} / \delta_{0}\right)^{4}}{48+0.8\left(r_{o} / \delta_{0}\right)^{4}}=1.019
$$

where, $r_{0}$. is the radius of wire and $r_{0}=0.255 \mathrm{~mm}$.

The influence of the proximity effect of the secondary winding is calculated by

$$
\Delta_{s}=\frac{d_{s}}{\delta_{0}}=\frac{0.15}{0.26}=0.577, \quad p_{s}=4
$$

where, $d_{S}$ is the diameter of wire. The ratio of secondary AC resistance and DC resistance is $R_{a c_{-} S} / R_{d c_{-} S}=1.2$. Power loss and the efficiency of transformer are shown in Table 5. It is worth noting the efficiency of the transformer is calculated under the condition of resonant frequency and full power. 
Table 5. Loss Calculations.

\begin{tabular}{ccc}
\hline Symbol & Description & Value \\
\hline$P_{\max }$ & Allowed Maximum power loss & $4.9 \mathrm{~W}$ \\
$P_{c u \_d c}$ & DC winding loss & $1.56 \mathrm{~W}$ \\
$P_{c u \_c c}$ & AC winding loss & $1.74 \mathrm{~W}$ \\
$P_{f e}$ & Core loss & $1.94 \mathrm{~W}$ \\
$P_{L a}$ & The loss of SCI & $1.1 \mathrm{~W}$ \\
$P_{t o l}$ & Total loss & $4.78 \mathrm{~W}$ \\
$\eta$ & Transformer efficiency & $99.37 \%$ \\
\hline
\end{tabular}

\subsection{Experimental Results}

The designed transformer is applied to $720 \mathrm{~W}$ LLC resonant converter charger. The experimental waveforms under different operating conditions are given in Figure 14. The operating waveform of LLC resonant converter at the initial stage of constant current charging is given in Figure 14a. The output power is $608 \mathrm{~W}$ and the operating frequency is $98.1 \mathrm{kHz}$, which is higher than the resonant frequency. The phase-shifting angle of the SCI is $\alpha=\pi / 2$, and the current of the SCI branch $i_{A B}$ is triangular wave. At this time, the magnetizing inductance of the resonant tank is small, the $\mathrm{K}$ value is also small, which helps reducing the switching frequency range.

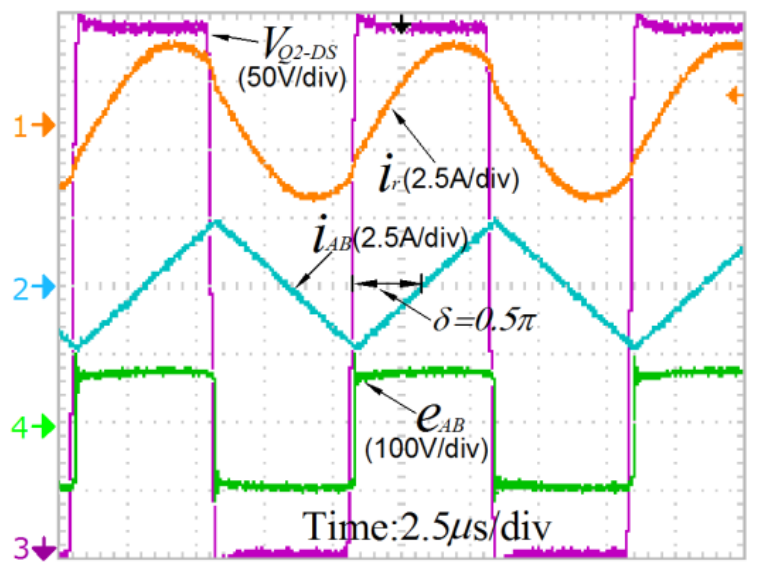

(a)

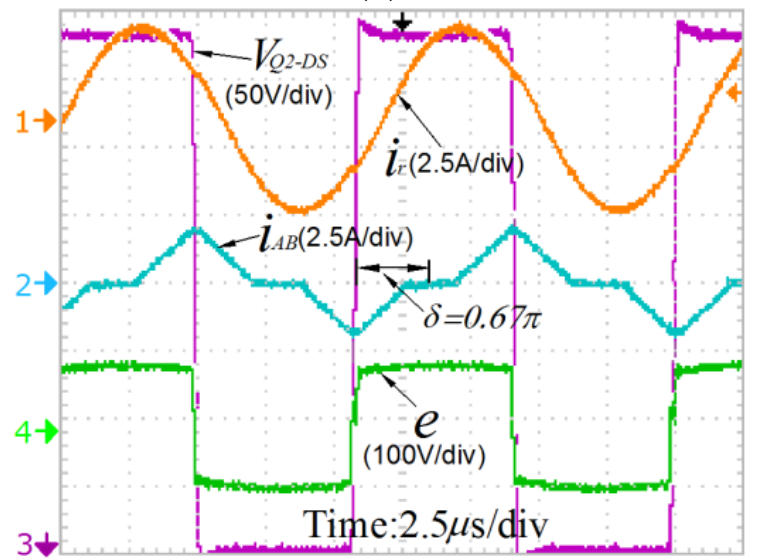

(b)

Figure 14. Cont. 


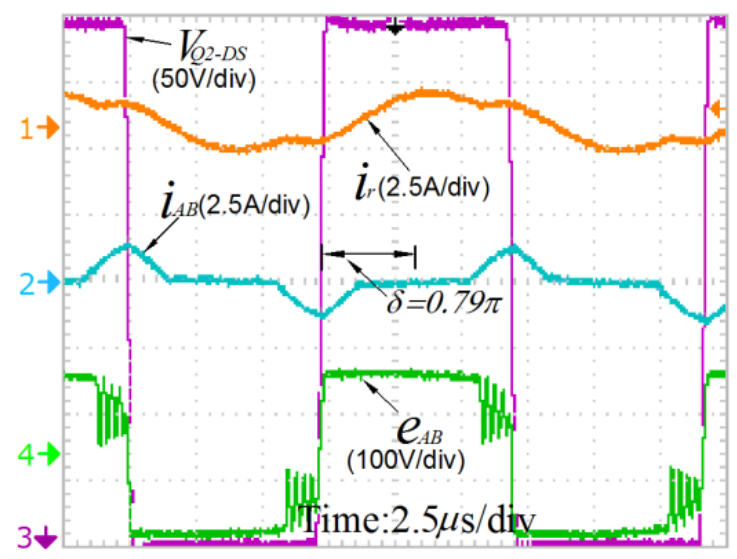

(c)

Figure 14. Experimental waveforms. (a) Constant Current Stage with $V_{o}=76 \mathrm{~V}$ and $I_{o}=8 \mathrm{~A}(\alpha=0.5 \pi)$; (b) Constant Power Stage with $V_{o}=90 \mathrm{~V}$ and $I_{o}=8 \mathrm{~A}(\alpha=0.67 \pi)$; (c) Constant Voltage Stage with $V_{o}=96 \mathrm{~V}$ and $I_{o}=2.5 \mathrm{~A}(\alpha=0.79 \pi)$.

Figure 14b shows the working waveform of LLC resonant converter at the constant power charging stage. The output power is $720 \mathrm{~W}$ and the operating frequency is $84.8 \mathrm{kHz}$, near the resonant frequency. The phase-shifting angle of the switch-controlled inductor is $\alpha \approx 0.67 \pi$, and the magnetizing current of the transformer is relatively decreased, which reduces the conduction loss.

Figure 14c shows the working waveform of LLC resonant converter at constant voltage charging stage. The output power is $240 \mathrm{~W}$, the operating frequency is $70.2 \mathrm{kHz}$, which is lower than the resonant frequency, and the output power is smaller. The phase-shifting angle of the switch-controlled inductor is $\alpha \approx 0.79 \pi$, the magnetizing current of the transformer is decreased further and the light load efficiency is improve effectively.

The experiment results comparison of the transformer temperature after operating under a full load for $1 \mathrm{~h}$ is shown in Figure 15. The temperature rise of the proposed transformer is $65.2{ }^{\circ} \mathrm{C}$. Experiments show that the transformer works in an ideal temperature rise range. In addition, the conventional transformer with air gap is applied to the same resonator circuit, the transformer retaining wall interval $d$ is $4.3 \mathrm{~mm}$, the temperature rise is measured as $83.8^{\circ} \mathrm{C}$. Moreover, the temperature rise of the secondary winding near the air-gap is the highest, which is $14.9^{\circ} \mathrm{C}$ higher than the proposed transformer.

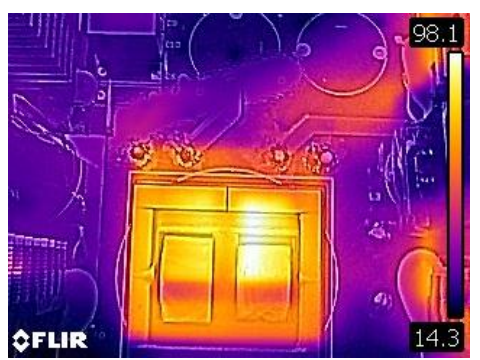

(a)

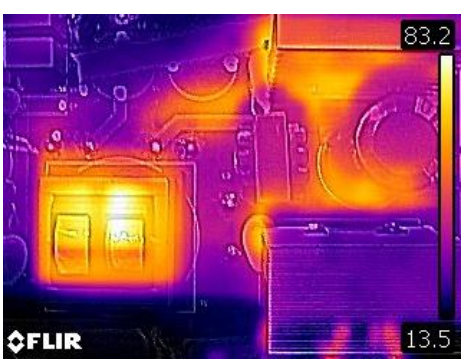

(b)

Figure 15. Temperature rise comparison. (a) The conventional scheme; (b) The proposed scheme.

Corresponding to Figure 14, the measured loss of the proposed transformer under $100 \%, 80 \%$, and 33\% loads are compared with those of the conventional LLC transformer, as shown in Figure 16. Experimental results show that the proposed design scheme is more efficient to reduce the temperature rise of magnetic components. 


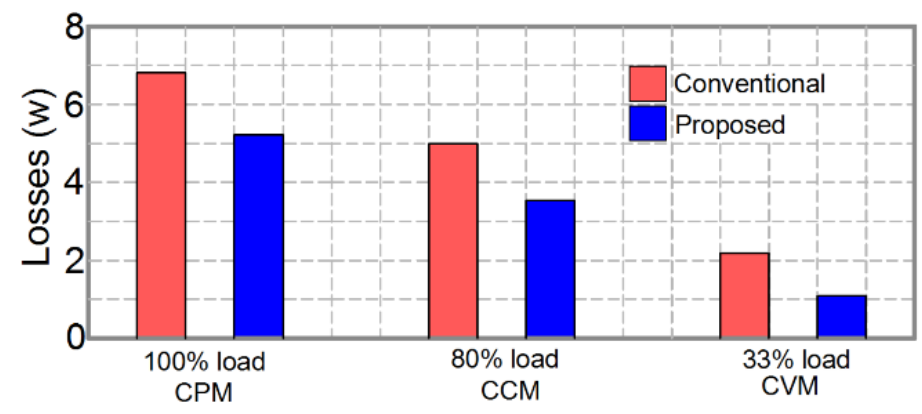

Figure 16. Measured loss comparison.

Figure 17 shows the efficiency comparison between the proposed LLC resonant converter charger and the conventional LLC resonant converter charger $(k=5)$ in the whole charging process. At the left of point $A$, the operating frequency is higher than resonant frequency. The proposed transformer magnetizing inductance can be dynamically adjusted, which not only improves the efficiency of the transformer itself, but also effectively reduces the conduction loss and switching loss of the LLC resonant converter. Hence, the comprehensive efficiency can be improved.

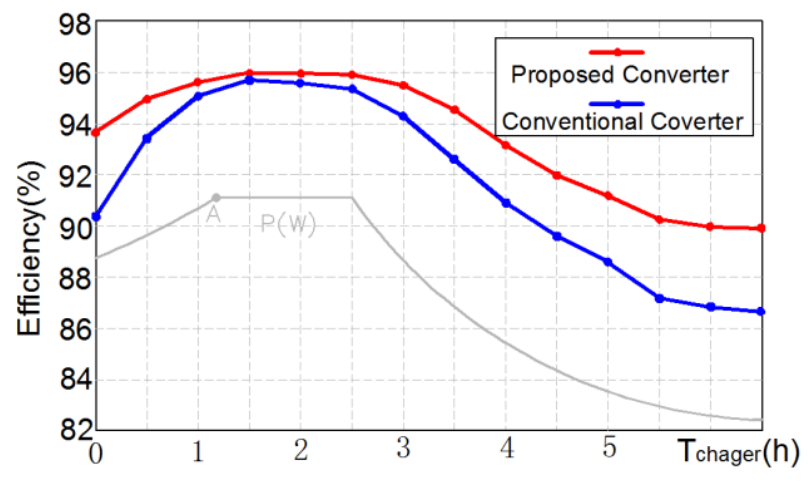

Figure 17. Efficiency comparison in the whole charging process.

\section{Conclusions}

Aiming at the relatively low efficiency problem of the conventional integrated transformer in LLC resonant converter, a controlled magnetizing inductance resonant transformer suitable for high power LLC resonant converter is proposed. The proposed transformer mainly removes the influence of fringing effect, improving the efficiency of the transformer, and the temperature rise of transformer. The experimental results show that the temperature rise of the transformer is reduced by $14.9{ }^{\circ} \mathrm{C}$ compared with the conventional scheme. Moreover, according to the change of charging process (output voltage and equivalent load impedance changes), the magnetizing inductance of proposed transformer can be dynamically adjusted to reduce the conduction loss and switching loss of the LLC resonant converter. Therefore, the comprehensive efficiency of the whole charging process can be improved further, especially at the initial charging stage and at the charging end (light load).

Author Contributions: All the authors conceived and designed the study. Z.Z., Q.X. performed the simulation and the experiment and wrote the manuscript with guidance from Y.D., Z.Z., Q.X., Y.D. and H.Y. reviewed the manuscript and provided valuable suggestions.

Funding: This work was supported by the National Natural Science Foundation of China (NSFC) under Grant No. 51807056.

Conflicts of Interest: The authors declare no conflict of interest. 


\section{References}

1. Deng, J.; Mi, C.C.; Ma, R.; Li, S. Design of LLC resonant converters based on operation-mode analysis for level two PHEV battery chargers. IEEE/ASME Trans. Mechatron. 2015, 20, 1595-1606. [CrossRef]

2. Demirel, I.; Erkmen, B. A Very Low-Profile Dual Output LLC Resonant Converter for LCD/LED TV Applications. IEEE Trans. Power Electron. 2014, 29, 3514-3524. [CrossRef]

3. Uno, M.; Kukita, A. Two-Switch Voltage Equalizer Using an LLC Resonant Inverter and Voltage Multiplier for Partially Shaded Series-Connected Photovoltaic Modules. IEEE Trans. Ind. Appl. 2015, 51, 1587-1601. [CrossRef]

4. Yang, B.; Lee, F.C.; Zhang, A.J.; Huang, G. LLC resonant converter for front end DC/DC conversion. In Proceedings of the Seventeenth Annual IEEE Applied Power Electronics Conference and Exposition, Dallas, TX, USA, 10-14 March 2002; pp. 1108-1112.

5. Wang, H.; Li, Z. A PWM LLC Type Resonant Converter Adapted to Wide Output Range in PEV Charging Applications. IEEE Trans. Power Electron. 2018, 33, 3791-3801. [CrossRef]

6. Jeong, Y.; Moon, G.W.; Kim, J.K. Analysis on half-bridge LLC resonant converter by using variable inductance for high efficiency and power density server power supply. In Proceedings of the 2017 IEEE Applied Power Electronics Conference and Exposition (APEC), Tampa, FL, USA, 26-30 March 2017; pp. 170-177.

7. Zhang, J.; Hurley, W.G.; Wölfle, W.H. Gapped Transformer Design Methodology and Implementation for LLC Resonant Converters. IEEE Trans. Ind. Appl. 2016, 52, 342-350. [CrossRef]

8. Saket, M.A.; Shafiei, N.; Ordonez, N. LLC Converters with Planar Transformers: Issues and Mitigation. IEEE Trans. Power Electron. 2017, 32, 4524-4542. [CrossRef]

9. Watanabe, T.; Kurokawa, F. Efficiency comparison between phase shift and LLC converters as power supply for information and communication equipment. In Proceedings of the 2015 IEEE International Telecommunications Energy Conference (INTELEC), Osaka, Japan, 18-22 October 2015; pp. 1-5.

10. Kim, E.S.; Kang, C.H.; Hwang, I.G.; Lee, Y.S.; Huh, D.Y. LLC Resonant Converter Using A Planar Transformer with New Core Shape. In Proceedings of the 2014 IEEE Applied Power Electronics Conference and Exposition-APEC 2014, Fort Worth, TX, USA, 16-20 March 2014; pp. 3374-3377.

11. Yamamoto, T.; Bu, Y.; Mizuno, T.; Yamaguchi, Y.; Kano, T. Loss Reduction of Transformer for LLC Resonant Converter Using a Magneto plated Wire. In Proceedings of the 2016 19th International Conference on Electrical Machines and Systems (ICEMS), Chiba, Japan, 13-16 November 2016; pp. 1-6.

12. Kang, B.G.; Park, C.S.; Chung, S.K. Integrated transformer using magnetic sheet for LLC resonant converter. Electron. Lett. 2014, 50, 770-771. [CrossRef]

13. Stadler, A.; Gulden, C. Copper Losses of Litz-Wire Windings Due to an Air Gap. In Proceedings of the 2013 15th European Conference on Power Electronics and Applications (EPE), Lille, France, 2-6 September 2013; pp. $1-7$.

14. Zhang, J.; Hurley, W.G.; Wölfle, W.H. Design of the Planar Transformer in LLC Resonant Converters for Micro-grid applications. In Proceedings of the 2014 IEEE 5th International Symposium on Power Electronics for Distributed Generation Systems (PEDG), Galway, Ireland, 24-27 June 2014; pp. 1-7.

15. Yang, S.; Abe, S.; Shoyama, M. Design Consideration of Two Flat Transformers in a Low-Profile LLC Resonant Converter. In Proceedings of the 8th International Conference on Power Electronics-ECCE Asia, Jeju, Korea, 30 May-3 June 2011; pp. 854-859.

16. Yang, S.; Abe, S.; Shoyama, M. Design Consideration of Flat Transformer in LLC Resonant Converter for Low Core Loss. In Proceedings of the 2010 International Power Electronics Conference (IPEC), Sapporo, Japan, 21-24 June 2010; pp. 343-348.

17. Alabakhshizadeh, A.; Midtgård, O.M. Winding Loss Analysis and Optimization of an AC Inductor for a Galvanically Isolated PV Inverter. In Proceedings of the 2012 International Conference and Exposition on Electrical and Power Engineering, Iasi, Romania, 25-27 October 2012; pp. 705-708.

18. Alabakhshizadeh, A.; Midtgård, O.M. Air Gap Fringing Flux Reduction in a High Frequency Inductor for a Solar Inverter. In Proceedings of the 2013 IEEE 39th Photovoltaic Specialists Conference (PVSC), Tampa, FL, USA, 16-21 June 2013; pp. 2849-2852.

19. Huang, W.N.; Lee, S.H.; Chen, C.G. Light-Load Efficiency Improvement Strategy for LLC Resonant Converter Utilizing a Step-Gap Transformer. In Proceedings of the 2014 International Power Electronics Conference, Hiroshima, Japan, 18-21 May 2014; pp. 1734-1737. 
20. Hua, C.C.; Fang, Y.H.; Lin, C.W. LLC resonant converter for electric vehicle battery chargers. IET Power Electron. 2016, 9, 2369-2376. [CrossRef]

21. Gu, W.J.; Harada, K. A new method to regulate resonant converters. IEEE Trans. Power Electron. 1998, 3, 430-439.

22. Musavi, F.; Craciun, M.; Gautam, D.S.; Eberle, W.; Dunford, W.G. An LLC Resonant DC-DC Converter for Wide Output Voltage Range Battery Charging Applications. IEEE Trans. Power Electron. 2013, 28, 5437-5445. [CrossRef]

23. Hurley, W.G.; Wölfle, W.H. Transformers and Inductors for Power Electronics. Theory, Design and Applications; Wiley: London, UK, 2013.

(C) 2018 by the authors. Licensee MDPI, Basel, Switzerland. This article is an open access article distributed under the terms and conditions of the Creative Commons Attribution (CC BY) license (http://creativecommons.org/licenses/by/4.0/). 